ARTICLE

\title{
Highly sensitive active pixel image sensor array driven by large-area bilayer $\mathrm{MoS}_{2}$ transistor circuitry
}

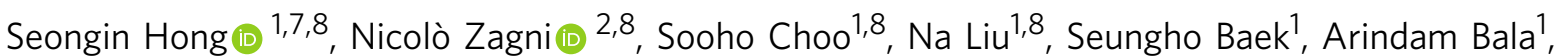
Hocheon Yoo $\mathbb{1}^{3}$, Byung Ha Kang ${ }^{4}$, Hyun Jae Kim (i) ${ }^{4}$, Hyung Joong Yun ${ }^{5}$, Muhammad Ashraful Alam ${ }^{6 凶} \&$ Sunkook Kim (1) ${ }^{1 凶}$

Various large-area growth methods for two-dimensional transition metal dichalcogenides have been developed recently for future electronic and photonic applications. However, they have not yet been employed for synthesizing active pixel image sensors. Here, we report on an active pixel image sensor array with a bilayer $\mathrm{MoS}_{2}$ film prepared via a two-step large-area growth method. The active pixel of image sensor is composed of 2D MoS switching transistors and $2 \mathrm{D} \mathrm{MoS}_{2}$ phototransistors. The maximum photoresponsivity $\left(R_{\mathrm{ph}}\right)$ of the bilayer $\mathrm{MoS}_{2}$ phototransistors in an $8 \times 8$ active pixel image sensor array is statistically measured as high as $119.16 \mathrm{~A} \mathrm{~W}^{-1}$. With the aid of computational modeling, we find that the main mechanism for the high $R_{\text {ph }}$ of the bilayer $\mathrm{MoS}_{2}$ phototransistor is a photo-gating effect by the holes trapped at subgap states. The image-sensing characteristics of the bilayer $\mathrm{MoS}_{2}$ active pixel image sensor array are successfully investigated using light stencil projection.

\footnotetext{
${ }^{1}$ School of Advanced Materials Science and Engineering, Sungkyunkwan University, Suwon, Republic of Korea. ${ }^{2}$ Department of Engineering "Enzo Ferrari" (DIEF), University of Modena and Reggio Emilia, Modena, Italy. ${ }^{3}$ Department of Electronic Engineering, Gachon University, Seongnam, Republic of Korea. ${ }^{4}$ School of Electrical and Electronic Engineering, Yonsei University, Seoul, Republic of Korea. ${ }^{5}$ Research Center for Materials Analysis, Korea Basic Science Institute (KBSI), Daejeon, Republic of Korea. ${ }^{6}$ School of Electrical and Computer Engineering, Purdue University, West Lafayette, Indiana, USA. ${ }^{7}$ Department of Electrical and Computer Engineering, The University of Texas at Austin, Austin, Texas 78758, USA. ${ }^{8}$ These authors contributed equally: Seongin Hong,

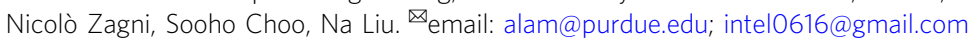


T wo-dimensional (2D) transition metal dichalcogenides (TMDs) such as molybdenum disulfide $\left(\mathrm{MoS}_{2}\right)$, molybdenum diselenide $\left(\mathrm{MoSe}_{2}\right)$, tungsten disulfide $\left(\mathrm{WS}_{2}\right)$, and tungsten diselenide $\left(\mathrm{WSe}_{2}\right)$ have been extensively studied as nextgeneration semiconducting materials due to their attractive electrical and optical properties ${ }^{1-9}$. However, although the TMD flakes obtained via mechanical exfoliation exhibit unique properties, their use in large-scale practical applications is difficult due to their low reproducibility and large property variations ${ }^{10-17}$. By contrast, various large-area growth methods for $2 \mathrm{D}$ TMDs have been developed for future electronic and photonic applications ${ }^{18-25}$. Choi et al. ${ }^{22}$ reported a full-color active-matrix organic lightemitting diode display based on large-area $\mathrm{MoS}_{2}$ synthesized via metal-organic chemical vapor deposition (CVD). Zhang et al. ${ }^{23}$ reported inverter arrays based on wafer-scale $\mathrm{MoS}_{2}$ synthesized via atomic layer deposition (ALD). Choi et al. ${ }^{24}$ reported a curved single photodetector array based on $\mathrm{MoS}_{2}$-graphene synthesized via CVD. Large-scale growth methods for TMDs have been reported, but they have not yet been employed to synthesize active pixel image sensors, which are integrated circuits consisting of photodetectors and active transistors that can detect the incident image light and convert it into digital image signals ${ }^{26-28}$.

In this study, we report on an active pixel image sensor array with a large-area bilayer $\mathrm{MoS}_{2}$ film, which was directly synthesized on a $\mathrm{SiO}_{2} / \mathrm{Si}$ substrate via a two-step growth method consisting of $\mathrm{MoS}_{2}$ sputtering (first step) and sulfurization (second step) without any transfer process. The circuitry in $8 \times 8$ active pixel image sensor array consists of switching transistors and phototransistors. The phototransistor used as a photodetector in the active pixel image sensor achieves a remarkably high photoresponsivity $\left(R_{\mathrm{ph}}\right)$ and signal-to-noise ratio (SNR). The main mechanism responsible for the high photoresponsivity of the bilayer $\mathrm{MoS}_{2}$ phototransistor is the photogating (PG) effect induced by light-generated holes trapped at subgap states. This explanation is supported by spectroscopic analysis and by numerical device simulations. The simulations highlight the correlation between threshold voltage $\left(V_{\mathrm{th}}\right)$ shift and high $R_{\mathrm{ph}}$ when including subgap states near the valence band edge. Moreover, both the 64 switching transistors and the 64 phototransistors based on homogeneous semiconductor (i.e., bilayer $\mathrm{MoS}_{2}$ ) in the $8 \times 8$ active pixel array are systematically investigated. It is revealed that the 64 individual pixels exhibit desired electrical and optical properties and high uniformity. Finally, we demonstrate the image-sensing characteristics of the active pixel image sensor array using light stencil projection. The proposed active pixel image sensor array can potentially be used for future image-sensing applications, such as ultra-thin image sensors, transparent image sensors, artificial-intelligence photosensors, and selective light-detecting imagers ${ }^{29-32}$.

\section{Results}

Structure design of bilayer $\mathrm{MoS}_{2}$ image sensor array. The active pixel image sensor array with a large-area bilayer $\mathrm{MoS}_{2}$ film and its pixel configuration are schematically and photographically illustrated in Fig. 1a-c and Fig. 1d-f, respectively. The designed device is composed of an $8 \times 8$ pixel array, in which the individual pixels have an opaque top-gate switching transistor and transparent top-gate phototransistor. The opaque top-gate electrode of the switching transistor can completely block the incident light, enabling the pixel selection operation without the influence of the light. By contrast, the transparent top-gate electrode of the phototransistor can successfully transmit the incident light, enabling the generation of electron-hole pairs in the $\mathrm{MoS}_{2}$ channel. Figure $1 \mathrm{~g}$ shows the equivalent circuit of a pixel in the active pixel image sensor array. The phototransistor was integrated into the pixel as a photodetector instead of a photodiode, which is typically used in active pixel image sensors, resulting in a higher photoresponsivity and SNR.

Fabrication process of bilayer $\mathrm{MoS}_{2}$ image sensor array. The presented architecture of the active pixel image sensor array was formed using a thin film process, owing to the atomically thin bilayer $\mathrm{MoS}_{2}$ film synthesized directly on a $\mathrm{SiO}_{2} / \mathrm{Si}$ substrate without any transfer process. Figure $2 \mathrm{a}$ shows the fabrication process of the pixel unit cell. The bilayer $\mathrm{MoS}_{2}$ film was directly synthesized on the cleaned $\mathrm{SiO}_{2} / \mathrm{Si}$ substrate via a two-step growth method. The synthesized bilayer $\mathrm{MoS}_{2}$ film was patterned via $\mathrm{O}_{2}$ reactive ion etching using a photoresist mask for channel isolation. Titanium/Au $(10 / 50 \mathrm{~nm})$ as the source/drain (S/D) electrodes were deposited and patterned using an electron-beam evaporator and photolithography via a lift-off technique. Aluminium oxide $(80 \mathrm{~nm})$ was deposited as the gate insulator via ALD. Subsequently, transparent top-gate electrodes (i.e., indium zinc oxide, IZO) and opaque top-gate electrodes (i.e., Au) were deposited via sputtering and e-beam evaporation, and patterned via photolithography with wet-etching and lift-off techniques, respectively. The details of the device fabrication are presented in the "Methods" section.

Synthesis of bilayer $\mathbf{M o S}_{\mathbf{2}}$ film. The two-step growth method consisting of radiofrequency (RF) magnetron sputtering and thermal CVD is schematically illustrated in Fig. 2b, c. $\mathrm{A} \mathrm{MoS}_{2}$ film was deposited on a $\mathrm{SiO}_{2} / \mathrm{Si}$ substrate via $\mathrm{RF}$ magnetron sputtering and then loaded into a CVD chamber for sulfurization. Hydrogen sulfide gas was used as a sulfur precursor in the sulfurization of sputtering deposited $\mathrm{MoS}_{2}$, which could cause a homogeneous reaction. The temperature profile and gas injection conditions as a function of time are shown in Fig. 2d. The $\mathrm{MoS}_{2}$ film synthesized via the two-step growth method shows a uniform color, indicating thickness uniformity of the $\mathrm{MoS}_{2}$ film on $\mathrm{SiO}_{2} / \mathrm{Si}$ substrate with the diagonal length of $\sim 9.57 \mathrm{~cm}$ (Fig. 3a).

Characterization of bilayer $\mathrm{MoS}_{2}$ film. High-resolution transmission electron microscopy (TEM) analysis was performed to explore the crystalline structure of the $\mathrm{MoS}_{2}$ film. As shown in Fig. 3b, the morphology of the $\mathrm{MoS}_{2}$ film appears smooth and consists of several grains. The corresponding fast Fourier transform pattern shows numerous spots forming halo rings, indicating that the $\mathrm{MoS}_{2}$ film has several random crystallite orientations ${ }^{33}$. According to Supplementary Fig. 1, the grain size is estimated to be $5-15 \mathrm{~nm}$, indicating a high density of the grain boundaries. The layer number of the $\mathrm{MoS}_{2}$ film was determined via the crosssectional TEM image shown in Fig. 3c. The $\mathrm{MoS}_{2}$ film consists of two layers, with an average interlayer spacing of $\sim 0.6 \mathrm{~nm}$, which is consistent with the theoretical and experimental values ${ }^{34}$.

The $\mathrm{MoS}_{2}$ film was characterized using Raman spectroscopy to investigate its crystallinity and number of layers. In Fig. $3 \mathrm{~d}$, the Raman spectrum shows two bands at 384.8 and $405.2 \mathrm{~cm}^{-1}$, which correspond to the in-plane $\left(E^{1}{ }_{2 \mathrm{~g}}\right)$ and out-of-plane $\left(A_{1 \mathrm{~g}}\right)$ vibration modes, respectively ${ }^{35}$. As the two modes exhibit a welldefined dependence on the number of layers, the frequency difference $(\Delta k)$ between the two modes is $20.4 \mathrm{~cm}^{-1}$, indicating a bilayer $\mathrm{MoS}_{2}$ film ${ }^{36}$. The Raman results are consistent with those of mechanically exfoliated $\mathrm{MoS}_{2}$, which indicates the high crystallinity of our $\mathrm{MoS}_{2}$ film grown via the two-step method. The thickness of the $\mathrm{MoS}_{2}$ film was also characterized using atomic force microscopy shown in Supplementary Fig. 2. The film thickness is estimated as $\sim 1.3 \mathrm{~nm}$, corresponding to a typical bilayer $\mathrm{MoS}_{2}{ }^{37}$. Photoluminescence (PL) measurement was used to investigate the optical quality of the $\mathrm{MoS}_{2}$ film. The PL 


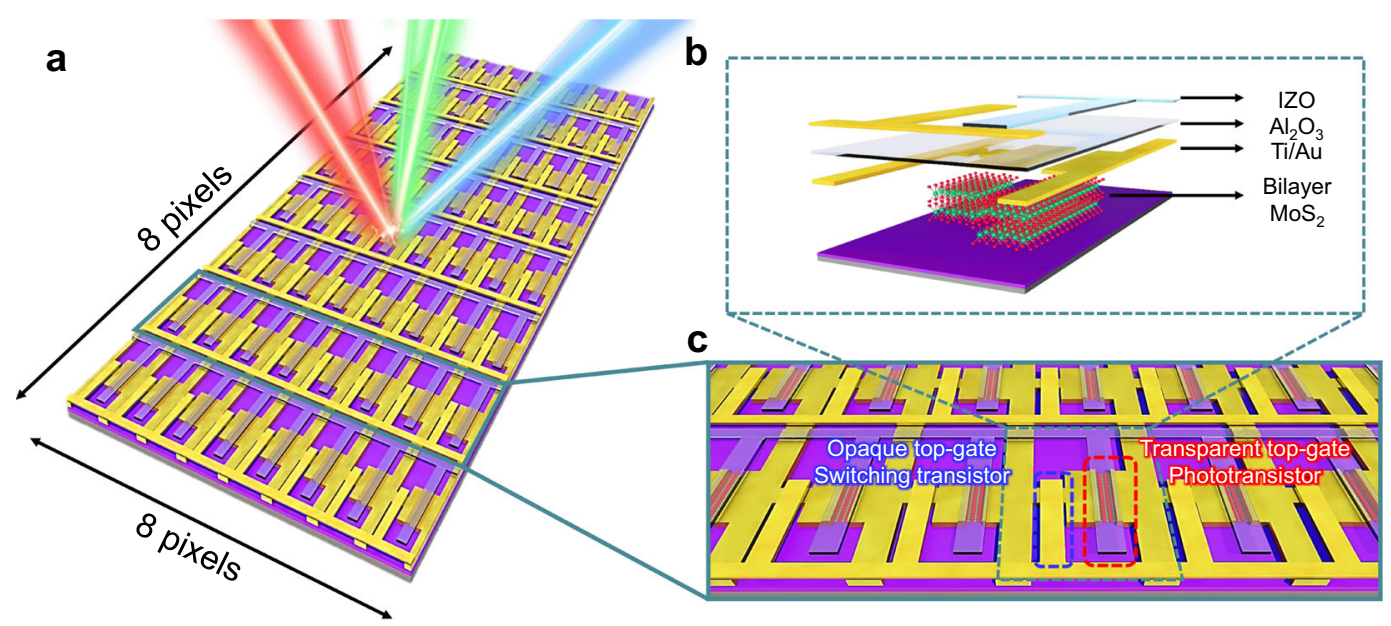

d

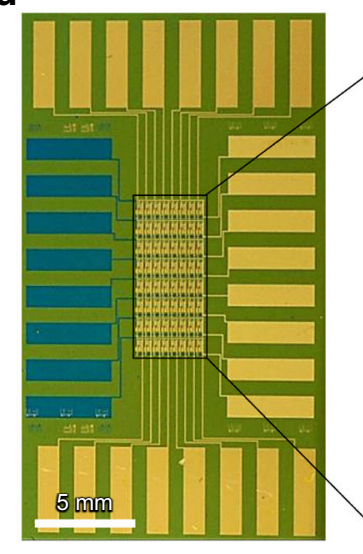

e

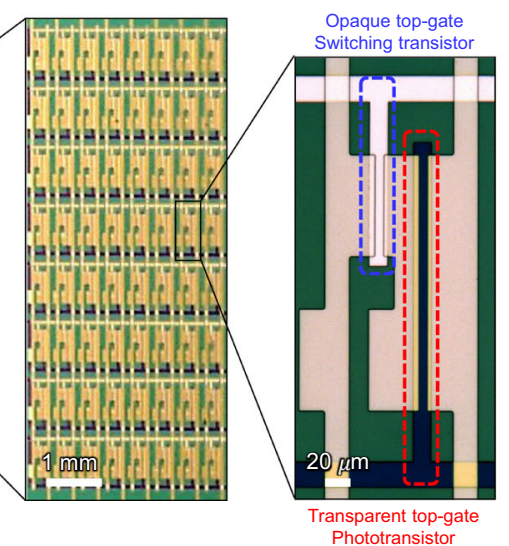

g

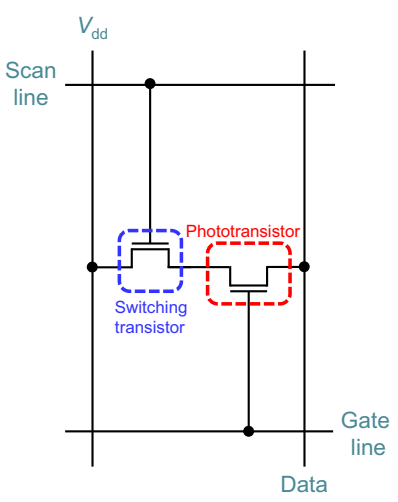

Fig. 1 Bilayer $\mathbf{M o S}_{\mathbf{2}}$ image sensor array. a Schematic illustration of an $8 \times 8$ image sensor array based on bilayer MoS 2 . b Cross-section of a pixel consisting of IZO, $\mathrm{Al}_{2} \mathrm{O}_{3}, \mathrm{Ti} / \mathrm{Au}$, and bilayer $\mathrm{MoS}_{2}$. $\mathbf{c}$ Image sensor array structure design composed of opaque top-gate (Ti/Au electrodes) switching transistor and transparent top-gate (IZO electrodes) phototransistor. $\mathbf{d}$ Low- and $\mathbf{e}$ high-magnification photograph of the $8 \times 8$ image sensor array based on bilayer $\mathrm{MoS}_{2}$. $\mathbf{f}$ Optical microscope image of a pixel composed of opaque top-gate switching transistor (blue-dashed line) and transparent top-gate phototransistor (red-dashed line). $\mathbf{g} \mathrm{A}$ pixel circuit diagram of proposed image sensor array. $V_{\mathrm{dd}}$ is drain supply voltage.

spectrum exhibits two peaks at $\sim 663$ and $617 \mathrm{~nm}$, corresponding to the $\mathrm{A} 1$ and $\mathrm{B} 1$ direct excitonic transitions at 1.97 and $2.11 \mathrm{eV}$, respectively (Fig. 3e). This indicates that the PL peaks originate from the intrinsic electronic structure of the grown $2 \mathrm{H}-\mathrm{MoS}_{2}{ }^{38}$. Moreover, a randomly selected area of $30 \mu \mathrm{m} \times 30 \mu \mathrm{m}$ was subjected to Raman mapping. Figure $3 f$, g display the Raman mapping images of the intensity of the $E_{2 \mathrm{~g}}^{1}$ and $A_{1 \mathrm{~g}}$ modes centered at 383 and $404 \mathrm{~cm}^{-1}$, respectively. The uniform color contrast demonstrates the high crystallinity and thickness uniformity of our grown $\mathrm{MoS}_{2}$ film at a microscale.

The chemical composition of the synthesized bilayer $\mathrm{MoS}_{2}$ film was studied using X-ray photoelectron spectroscopy (XPS). In Fig. $3 \mathrm{~h}$, the core-level spectrum of Mo $3 d$ exhibits two strong peaks at 228.9 and $232.0 \mathrm{eV}$ corresponding to $\mathrm{Mo}^{4+} 3 d_{5 / 2}$ and $\mathrm{Mo}^{4+} 3 d_{3 / 2}$ (Mo-S bonding), respectively, and two weak peaks at 232.4 and $235.5 \mathrm{eV}$ corresponding to $\mathrm{Mo}^{6+} 3 d_{5 / 2}$ and $\mathrm{Mo}^{6+} 3 d_{3 / 2}$ (Mo-O bonding), respectively ${ }^{39,40}$. The Mo-O bonding is attributed to $\mathrm{MoO}_{3}$, which is contained in the $\mathrm{MoS}_{2}$ target. The atomic fraction of $\mathrm{Mo}^{6+} 3 d$ contained in the grown bilayer $\mathrm{MoS}_{2}$ is $\sim 10.55 \%$. Notably, no additional peak is observed at the low binding energy of $\sim 229.0 \mathrm{eV}$, which corresponds to metallic $1 \mathrm{~T}-\mathrm{MoS}_{2}$ or metal $\mathrm{Mo}^{41}$. This indicates that the sputtered $\mathrm{MoS}_{2}$ is fully sulfurized to form $2 \mathrm{H}-\mathrm{MoS}_{2}$ without a $1 \mathrm{~T}-\mathrm{MoS}_{2}$ component. In addition, Fig. 3i shows two $\mathrm{S} 2 p$ peaks at 161.8 and $163.0 \mathrm{eV}$, corresponding to the doublet of $S^{2-} 2 p_{3 / 2}$ and $S^{2-} 2 p_{1 / 2}$, respectively, which further confirms the $2 \mathrm{H}-\mathrm{MoS}_{2}$ crystal structure. The calculated atomic ratio between $\mathrm{S}^{2-}$ $2 p$ and $\mathrm{Mo}^{4+} 3 d$ is $\sim 2.14$, indicating a S-rich $\mathrm{MoS}_{2}$ with good crystallinity grown via the two-step method.

Ultraviolet photoelectron spectroscopy analysis was used to study the electronic structure of the synthesized $\mathrm{MoS}_{2}$ film under ultra-high vacuum using $\mathrm{He} \mathrm{I}$ as a monochromatic excitation source. The work function $(\Phi)$ can be calculated using $\Phi=h v-$ $W$, where $h v$ is the incident photon energy of $21.22 \mathrm{eV}(\mathrm{He} \mathrm{I})$ and $W$ is the spectral width extracted from the intersection of the slope of the secondary cut-off spectrum with the baseline (Fig. 3j). The measured work function for the $\mathrm{MoS}_{2}$ film is $4.40 \mathrm{eV}$, for which the value is consistent with the other reports ${ }^{42-45}$. In addition, the valence band maximum (VBM) can also be determined from the intersection of the slope of the first state from Fermi energy $\left(E_{\mathrm{F}}=0 \mathrm{eV}\right)$ with the baseline (Fig. $\left.3 \mathrm{k}\right)$. The extracted difference of energy between the $E_{\mathrm{F}}$ and VBM is 1.65 $\mathrm{eV}$. As previous reports, the few-layer $\mathrm{MoS}_{2}$ exhibit a bandgap from 1.29 to $1.9 \mathrm{eV}$ and it could be inferred that the synthesized $\mathrm{MoS}_{2}$ film was an $n$-type semiconductor ${ }^{46}$.

Electrical characteristics of $\mathrm{MoS}_{2}$ devices in the array. $I-V$ characteristics were measured for the 64 phototransistors and 

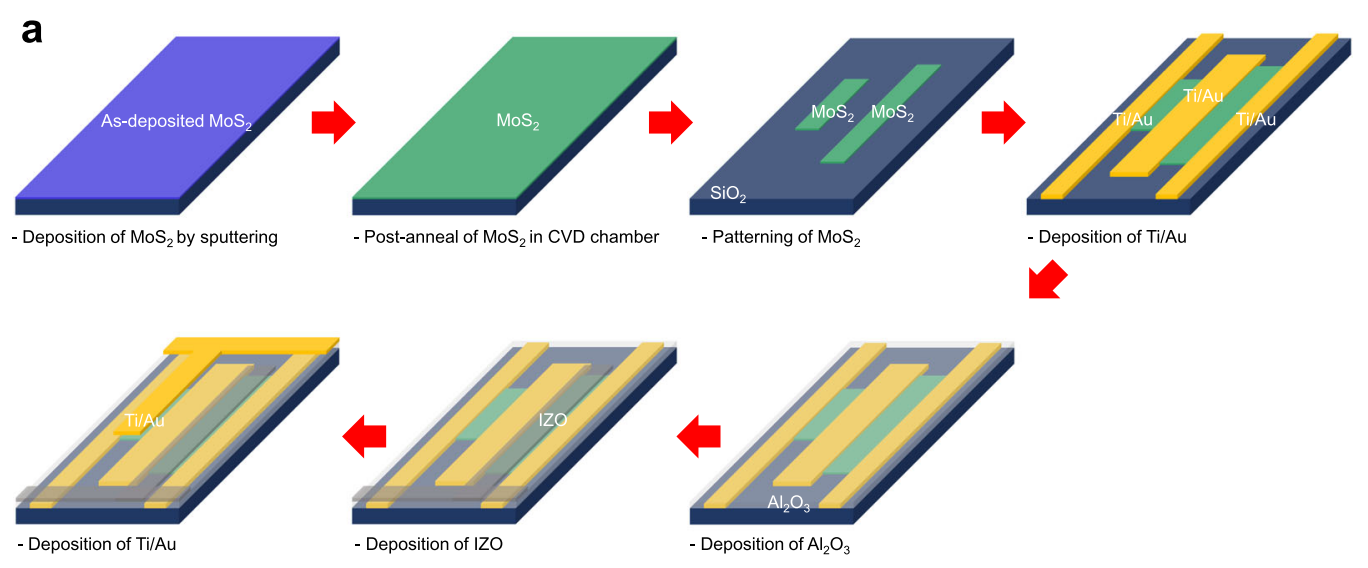

b

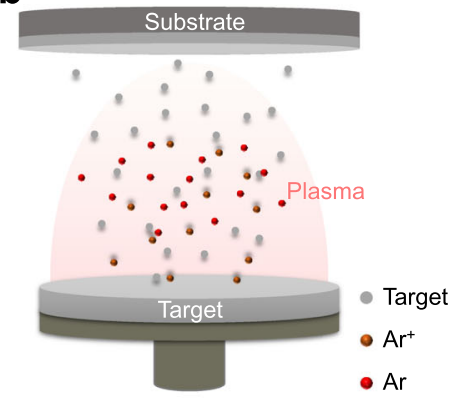

C

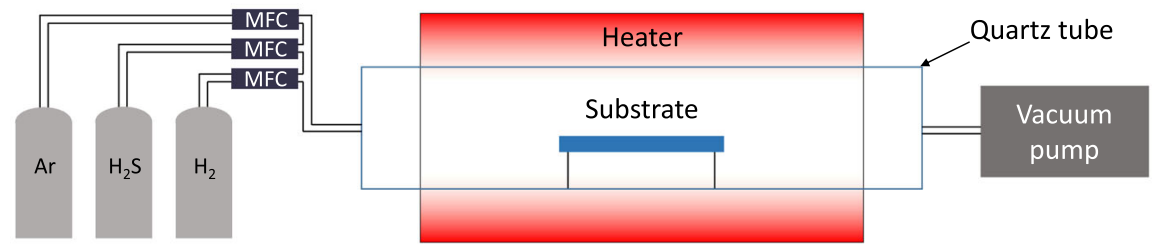

d

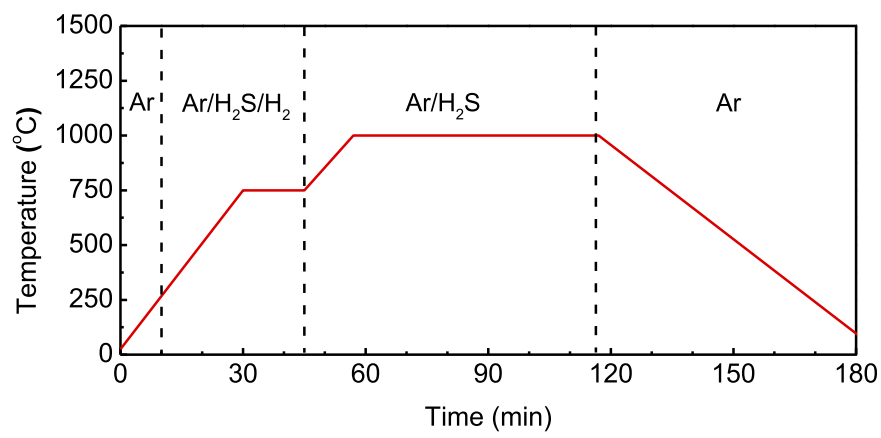

Fig. 2 Fabrication steps of the image sensor array based on bilayer $\mathbf{M o S}_{\mathbf{2}}$ film. a Fabrication process of a pixel unit cell in the proposed active pixel image sensor array with a bilayer $\mathrm{MoS}_{2}$ film. Synthesis of a bilayer $\mathrm{MoS}_{2}$ film using a two-step growth method: $\mathrm{MoS}_{2}$ sputtering (first step) and sulfurization in the CVD (second step). Schematic image of b RF magnetron sputtering and c CVD chamber composed of gas sources $\left(\mathrm{Ar}, \mathrm{H}_{2} \mathrm{~S}, \mathrm{H}_{2}\right)$, quartz tube, heater, and vacuum pump. MFC is mass flow controller. $\mathbf{d}$ Temperature profile and gas injection conditions for sulfurization as a function of time. The vertical dashed lines divide four regions with different gas atmospheres.

64 switching transistors in the $8 \times 8$ active pixel image sensor array, to investigate its electrical properties. Figure 4 a shows the transfer curves of a $\mathrm{MoS}_{2}$ phototransistor in the active pixel image sensor array, indicating typical $n$-channel behaviors with a current on/off ratio $\left(I_{\text {on }} / I_{\text {off }}\right)$ of $5.84 \times 10^{4}$ and a threshold voltage $\left(V_{\mathrm{th}}\right)$ of $-22.32 \mathrm{~V}$ at a drain voltage $\left(V_{\mathrm{ds}}\right)$ of $5 \mathrm{~V}$. Figure $4 \mathrm{~b}$ also shows the output characteristics of the phototransistor. The drain current $\left(I_{\mathrm{ds}}\right)$ exhibits a linear behavior at a low drain bias due to the good ohmic contacts between the bilayer $\mathrm{MoS}_{2}$ film and the S/ $\mathrm{D}$ electrodes $(\mathrm{Ti} / \mathrm{Au})$, and shows a fully saturated current at a high drain bias due to the velocity-saturated charge carriers. Supplementary Fig. 3 shows comparison with the transfer curves of our bilayer, few-layer, and multilayer $\mathrm{MoS}_{2}$ phototransistors under the back-gate and top-gate modulations. Bilayer $\mathrm{MoS}_{2}$ exhibits the best performance than the other number of layers of $\mathrm{MoS}_{2}$ grown by the two-step method. The electrical properties of $\mathrm{MoS}_{2}$ phototransistors show metallic as the number of layer increase over the bilayer. Under back-gate modulation, the bilayer $\mathrm{MoS}_{2}$ phototransistor shows improved electrical properties (red line) after $\mathrm{Al}_{2} \mathrm{O}_{3}$ passivation compared with that before $\mathrm{Al}_{2} \mathrm{O}_{3}$ passivation (black line). Under the top-gate modulation, the electrical properties (blue line) of the device were significantly improved compared with that in the case of the back-gate modulation with the $\mathrm{Al}_{2} \mathrm{O}_{3}$ passivation layer (red line), which are attributed to the $n$-type doping effect ${ }^{47,48}$ and the high- $k$ dielectric screening effect ${ }^{49,50}$ of the $\mathrm{Al}_{2} \mathrm{O}_{3}$ layer.

The statistical analysis of the electrical performance parameters of the 64 phototransistors in the active pixel image sensor array is summarized in Fig. 4c-e, which was obtained from the transfer curves of the 64 phototransistors as shown in Supplementary Fig. 4a. Figure 4c shows the distribution of the field-effect mobility $\left(\mu_{\mathrm{eff}}\right)$, which is calculated from the following equation: $\mu_{\text {eff }}=g_{\mathrm{m}} \frac{L_{\mathrm{c}}}{W_{\mathrm{c}} C_{\mathrm{ox}} V_{\mathrm{ds}}}$, where $g_{\mathrm{m}}$ is the transconductance, $L_{\mathrm{c}}$ and $W_{\mathrm{c}}$ are the length and width of the channel, respectively, $C_{\mathrm{ox}}$ is the capacitance of the gate insulator, and $V_{\mathrm{ds}}$ is the drain voltage. Furthermore, Fig. $4 \mathrm{~d}$, e show the distribution of the $V_{\text {th }}$ and $I_{\mathrm{on}} /$

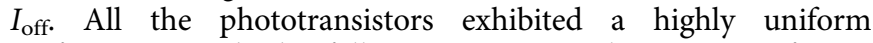
performance with the following average values: a $\mu_{\text {eff }}$ of 4.85 $\mathrm{cm}^{2} \mathrm{~V}^{-1} \mathrm{~s}^{-1}$, a $V_{\text {th }}$ of $-23.65 \mathrm{~V}$, and an $I_{\text {on }} / I_{\text {off }}$ of $3.88 \times 10^{4}$. The 64 switching transistors were quantitatively analyzed using the above method repeatedly. Details of the switching transistors are shown in Supplementary Figs. $4 \mathrm{~b}$ and 5. 
a

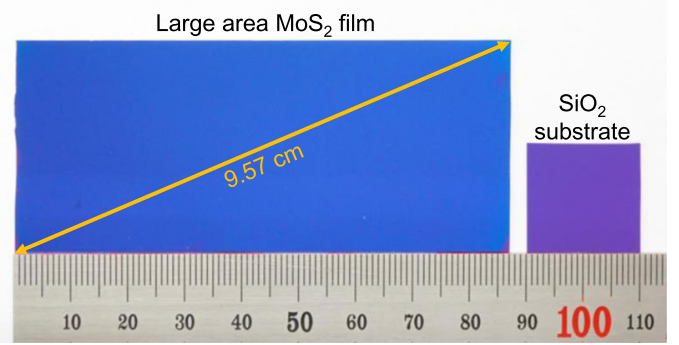

d

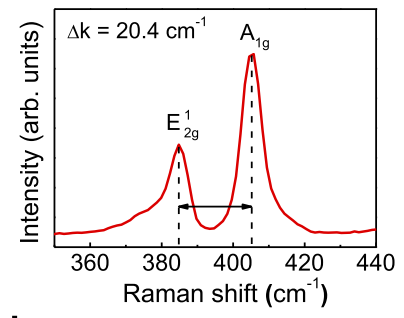

h

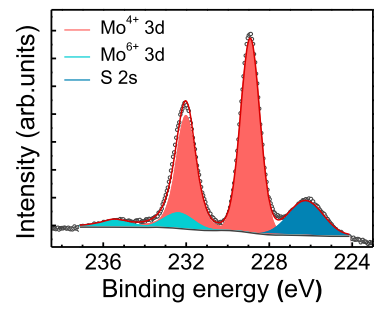

e

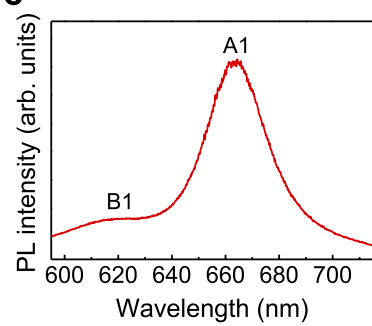

i

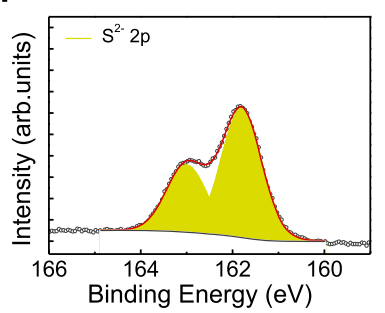

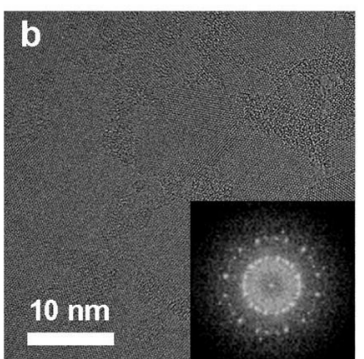

f

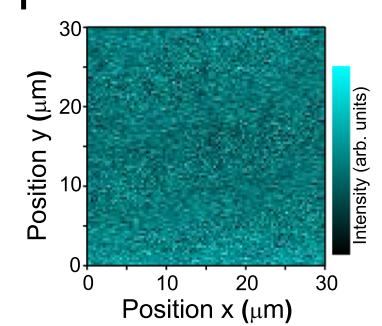

j

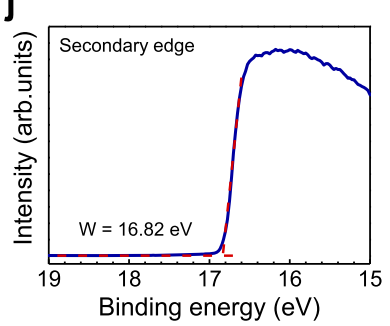

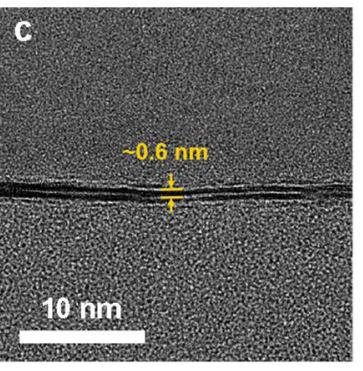

g

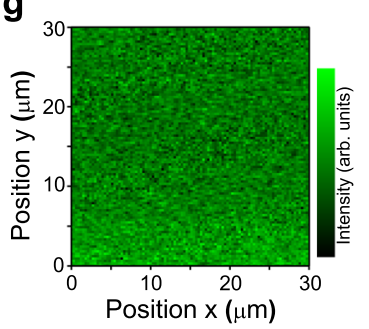

k

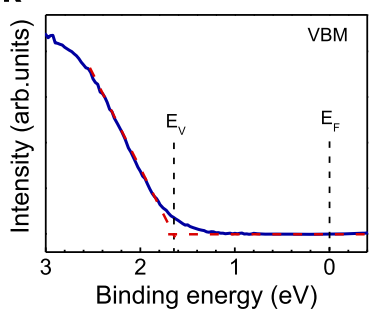

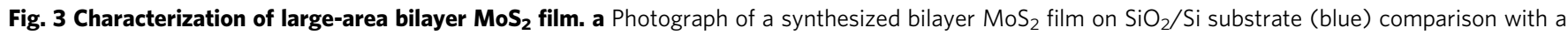
bare $\mathrm{SiO}_{2} / \mathrm{Si}$ substrate (purple), indicating the color changes for before and after $\mathrm{MoS}_{2}$ growth. b Plan-view and c cross-sectional TEM images of the bilayer $\mathrm{MoS}_{2}$ film. The inset of $\mathbf{b}$ is a FFT pattern corresponding to the TEM image. $\mathbf{d}$ Raman and e PL spectrum of the bilayer MoS film. $\mathbf{f}, \mathbf{g}$ Raman

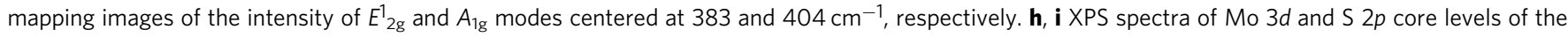
bilayer $\mathrm{MoS}_{2}$ film, respectively. $\mathbf{j}$ Secondary cut-off and $\mathbf{k}$ valence band spectra obtained by UPS analysis of bilayer MoS film.

Photoresponsive characteristics of $\mathrm{MoS}_{2}$ devices in the array. The proposed transparent top-gate phototransistor structure based on the bilayer $\mathrm{MoS}_{2}$ active layer and IZO top-gate electrode in Fig. 1 can easily detect the incident light because of the light transmitted through the transparent top-gate electrode, even using an opaque $\mathrm{SiO}_{2} / \mathrm{Si}$ substrate on which $\mathrm{MoS}_{2}$ is directly grown without additional and complex processes (i.e., transferring $\mathrm{MoS}_{2}$ onto glass or flexible substrate such as polyethylene terephthalate or polyimide films ${ }^{22,24,47}$, resulting in low productivity and high cost). Figure $5 \mathrm{a}-\mathrm{c}$ present the photoinduced transfer characteristics of the bilayer $\mathrm{MoS}_{2}$ phototransistor in the active pixel image sensor array under red, green, and blue (RGB) light illumination with an excitation wavelength $\left(\lambda_{\text {ex }}\right)$ of $638 \mathrm{~nm}$ $(\mathrm{R}), 532 \mathrm{~nm}(\mathrm{G})$, and $405 \mathrm{~nm}(\mathrm{~B})$ at various incident power densities $\left(P_{\text {inc }}\right)$ ranging from 0.1 to $3.2 \mathrm{~mW} \mathrm{~cm}^{-2}$. The photocurrent of the bilayer $\mathrm{MoS}_{2}$ phototransistor gradually increased with increasing $P_{\text {inc }}$. Figure $5 \mathrm{~d}-\mathrm{f}$ show the calculated photoresponsivity $\left(R_{\mathrm{ph}}\right)$, specific detectivity $\left(D^{*}\right)$, and photosensitivity $\left(S_{\mathrm{ph}}\right)$ as functions of excitation wavelength and incident power density, which are important figures of merit for phototransistors. The $R_{\mathrm{ph}}$ was extracted from the transfer characteristics in Fig. $5 \mathrm{a}-\mathrm{c}$, using the equation of $R_{\mathrm{ph}}=I_{\mathrm{ph}} / P_{\text {inc }}\left(\mathrm{A} \mathrm{W}^{-1}\right)$, where $I_{\mathrm{ph}}$ and $P_{\text {inc }}$ are the photocurrent and incident power density, respectively. The $D^{*}$ was obtained by the equation of $D^{*}$ $=\sqrt{A \Delta f} / \mathrm{NEP}=R \sqrt{A \Delta f} / i_{\mathrm{n}}$, where $A$ is the channel area, $\Delta f$ is the electrical bandwidth, NEP is the noise equivalent power, $i_{\mathrm{n}}$ is the noise current, and $R$ is the responsivity at the same measurement conditions as the noise current $\left(i_{\mathrm{n}}\right)^{51}$. The noise current, used to obtain the $D^{*}$, was measured by a lock-in amplifier (Supplementary Fig. 6). We obtained the $S_{\mathrm{ph}}$ defined as $S_{\mathrm{ph}}=I_{\mathrm{ph}} / I_{\text {dark }}$, where $I_{\text {dark }}$ is the dark current, extracted from the transfer curves of the $\mathrm{MoS}_{2}$ phototransistor. The maximum $R_{\mathrm{ph}}$ of $119.16 \mathrm{~A} \mathrm{~W}^{-1}$ and $D^{*}$ of $4.66 \times 10^{6} \mathrm{~cm} \mathrm{~Hz}^{1 / 2} \mathrm{~W}^{-1}$ were obtained under the condition of $\lambda_{\mathrm{ex}}=405 \mathrm{~nm}$ and $P_{\text {inc }}=0.1 \mathrm{~mW} \mathrm{~cm}^{-2}$ for the former and $\lambda_{\mathrm{ex}}=532 \mathrm{~nm}$ and $P_{\mathrm{inc}}=0.1 \mathrm{~mW} \mathrm{~cm}^{-2}$ for the latter. The maximum $S_{\mathrm{ph}}$ of 1173.44 was obtained at $\lambda_{\mathrm{ex}}=405 \mathrm{~nm}$ and $P_{\text {inc }}=3.2 \mathrm{~mW} \mathrm{~cm}^{-2}$. This is high $R_{\mathrm{ph}}$ reported for phototransistors with large-area synthesized $\mathrm{MoS}_{2}$ films as shown in Supplementary Fig. 7 and Table 1.

The main mechanism for the high $R_{\mathrm{ph}}$ of the bilayer $\mathrm{MoS}_{2}$ phototransistor is PG effect by the holes trapped at subgap states $^{33,52-55}$. The TEM and XPS analysis in Fig. 3 show that high density of grain boundaries, excess $\mathrm{S}$, and a small amount of $\mathrm{MoO}_{3}$ are present in the grown $\mathrm{MoS}_{2}$ film. Previous works report that structural defects in $\mathrm{MoS}_{2}$ itself can induce subgap states in the conduction and valence band ${ }^{56,57}$. In Fig. $3 \mathrm{k}$, a narrow distribution of states exists above the VBM in the bandgap. These band tail states near the valence band can capture the photogenerated holes, leading to the accumulation of positive charges and potential barrier lowering (i.e., PG effect). Injecting more electrons from the source by PG effect results in a significant enhancement of photoresponsivity in our $\mathrm{MoS}_{2}$ phototransistor. Supplementary Fig. 8 shows the details of PG effect on the bilayer $\mathrm{MoS}_{2}$ phototransistor through the extracted $V_{\text {th }}$ shift $\left(\triangle V_{\text {th }}\right)$ and $I_{\mathrm{ph}}$ as a function of $P_{\text {inc }}$. Supplementary Fig. $8 \mathrm{a}$ exhibits $\triangle V_{\mathrm{th}}-P_{\text {inc }}$ curves of the bilayer $\mathrm{MoS}_{2}$ 
a

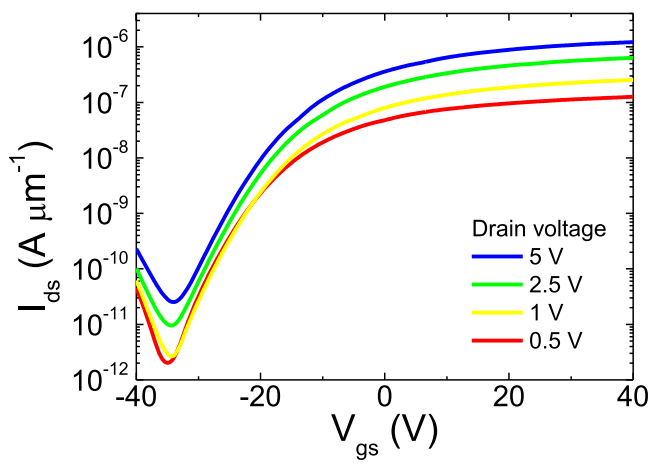

C

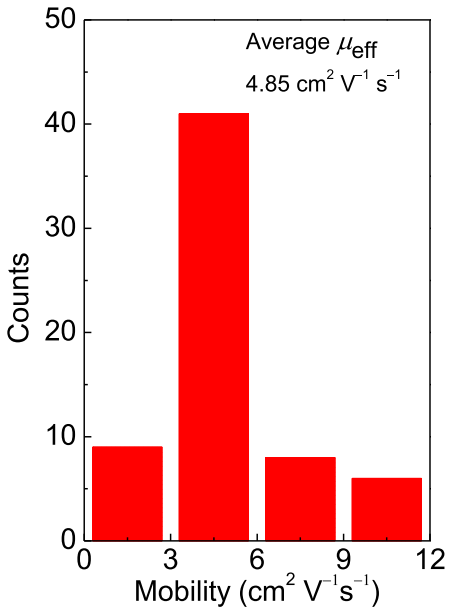

d

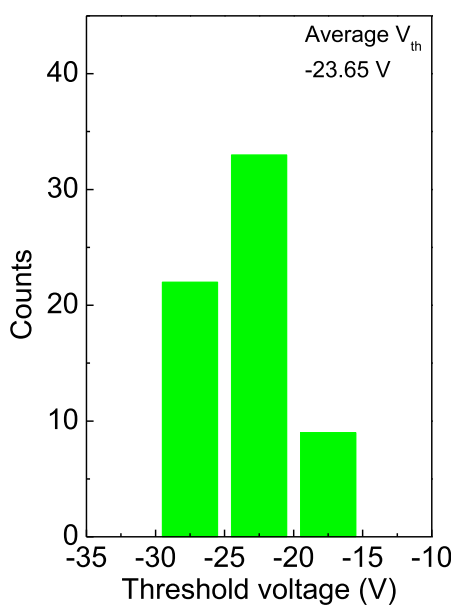

b

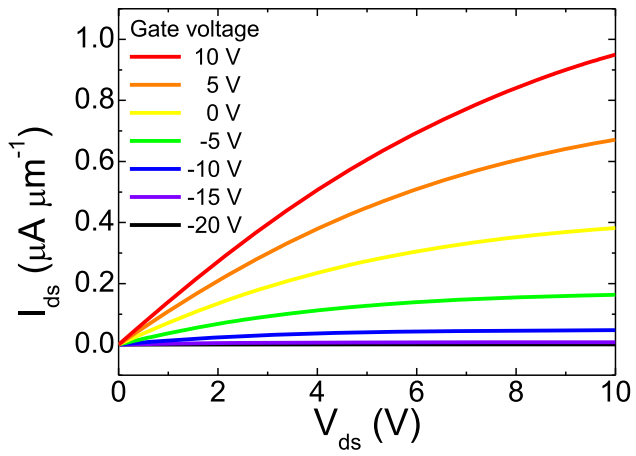

e

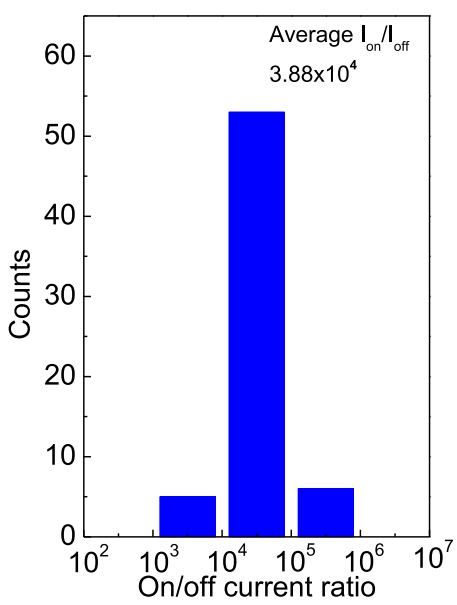

Fig. 4 Electrical characteristics and statistical analysis of phototransistors in bilayer $\mathbf{M o S}_{\mathbf{2}}$ image sensor array. a $V_{\mathrm{gs}}-l_{\mathrm{ds}} \mathrm{curves}_{\mathrm{r}}$ of $\mathrm{MoS}_{2}$ phototransistor at $V_{\mathrm{ds}}=0.5,1,2.5$, and $5 \mathrm{~V}$. $\mathbf{b} V_{\mathrm{ds}}-I_{\mathrm{ds}}$ curves of $\mathrm{MoS}_{2}$ phototransistor at $V_{\mathrm{gs}}$ from -20 to $10 \mathrm{~V}$ with the gap of $5 \mathrm{~V}$. Histograms of $\mathbf{c}$ field-effect mobility (average $\mu_{\text {eff }}=4.85 \mathrm{~cm}^{2} \mathrm{~V}^{-1} \mathrm{~s}^{-1}$ ), $\mathbf{d}$ threshold voltage (average $V_{\text {th }}=-23.65 \mathrm{~V}$ ), and $\mathbf{e}$ on/off current ratio $\left(\right.$ average $I_{\text {on }} / I_{\text {off }}=3.88 \times 10^{4}$ ) of the $64 \mathrm{MoS}_{2}$ phototransistors

phototransistor under RGB light illumination. As depicted in the inset of Supplementary Fig. 8a, holes trapped at subgap states act as a local gate, resulting in the negative shift of $V_{\text {th }}$ as the $P_{\text {inc }}$ increased from 0.1 to $3.2 \mathrm{~mW} \mathrm{~cm}^{-2}$. Supplementary Figs. $8 \mathrm{~b}-\mathrm{d}$ show the $I_{\mathrm{ph}}-P_{\text {inc }}$ curves of the device under RGB light illumination, respectively ${ }^{53}$. By sweeping the gate voltage $\left(V_{\mathrm{gs}}\right)$ from -40 to $40 \mathrm{~V}$, the slope of $I_{\mathrm{ph}}-P_{\text {inc }}$ (i.e., $\alpha$ : absorption coefficient) decreases, indicating that the dominant mechanism for $R_{\mathrm{ph}}$ high switches from photoconductive (PC) effect to PG effect $^{33,54}$. PC effect is that the conductivity of the channel is increased by the photogenerated electron-hole pairs, whereas PG effect is one of special PC effect resulted from accumulation of holes and conduction band lowering due to the defects, impurities, or multi-junction structure of channel materials. The PG effect affects similarly to the additional $V_{\mathrm{gs}}$, resulting in decrease of $\alpha$ as the $V_{\mathrm{gs}}$ increases.

Figure $5 \mathrm{~g}-\mathrm{i}$ show the photoswitching behaviors of the bilayer $\mathrm{MoS}_{2}$ phototransistor in the active pixel image sensor array under pulsed RGB light illumination with the frequency of $1 \mathrm{~Hz}$. The rise time $\left(\tau_{\mathrm{r}}\right)$ and fall time $\left(\tau_{\mathrm{f}}\right)$ were defined as the times taken for the current to change from $20 \%$ to $80 \%$ and from $80 \%$ to $20 \%$ of the maximum current, respectively ${ }^{58}$. The photoresponse speeds of the bilayer $\mathrm{MoS}_{2}$ phototransistor under RGB light illumination were $\tau_{\mathrm{r} \text { under R }}, \tau_{\mathrm{r} \text { under G }}, \tau_{\mathrm{r} \text { under B }}=44,85,119 \mathrm{~ms}$ and $\tau_{\mathrm{f}}$ under R , $\tau_{\mathrm{f}}$ under $\mathrm{G}, \tau_{\mathrm{f}}$ under $\mathrm{B}=41,52,122 \mathrm{~ms}$, respectively. $\tau_{\mathrm{r}}$ and $\tau_{\mathrm{f}}$ increase as $\lambda_{\mathrm{ex}}$ decreases due to the greater number of photogenerated charge carriers. Such a satisfactory photoresponse speed indicates that the bilayer $\mathrm{MoS}_{2}$ active pixel image sensor array has the potential for fast image sensing. However, the response time still needs to be improved for high-speed imaging due to the trade-off relationship between response time and responsivity. Therefore, to improve the response speed of our image sensor array, we investigated the photoswitching characteristics of the bilayer $\mathrm{MoS}_{2}$ phototransistor with gate pulse as shown in Supplementary Fig. 9. The $40 \mathrm{~ms}$ gate voltage pulse significantly enhanced the fall time from $104.8 \mathrm{~ms}$ to $22.99 \mathrm{~ms}$ due to the detrapping of the trapped holes at subgap states, enabling high-speed operation of the image sensor $29,59-61$. Supplementary Table 2 shows the comparison of the photoresponse speed without and with gate pulse. The bilayer $\mathrm{MoS}_{2}$ photodetector without a gate terminal in the same pixel was measured under the same conditions as in Fig. 5 to compare its photoresponsive characteristics with those of the proposed phototransistor (Supplementary Fig. 10). Consequently, compared with the photodetector without a gate terminal, the phototransistor showed significantly improved photoresponsivity, specific detectivity, and photosensitivity (i.e., SNR) by 14.01, 6.68, and 505.79 times, respectively.

Simulation results of bilayer $\mathrm{MoS}_{\mathbf{2}}$ phototransistor. To support the interpretation regarding the high $R_{\mathrm{ph}}$ of the bilayer $\mathrm{MoS}_{2}$ phototransistor being induced by the PG effect, numerical device simulations were carried out. The simulation framework is based on the drift-diffusion formalism (see "Simulation methods" for details of the simulation setup) with material and device 


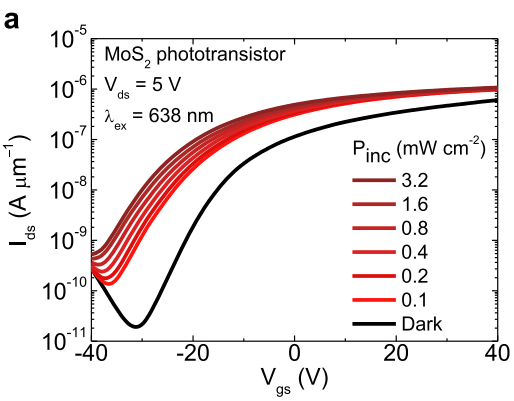

d

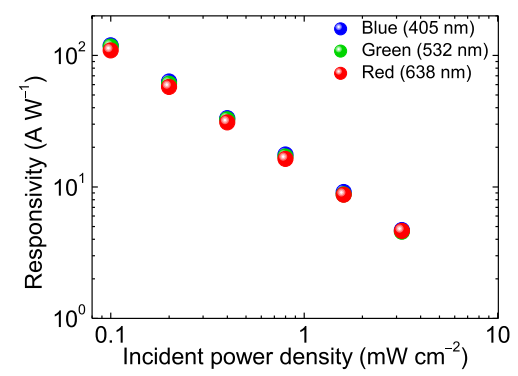

$\mathbf{9}$

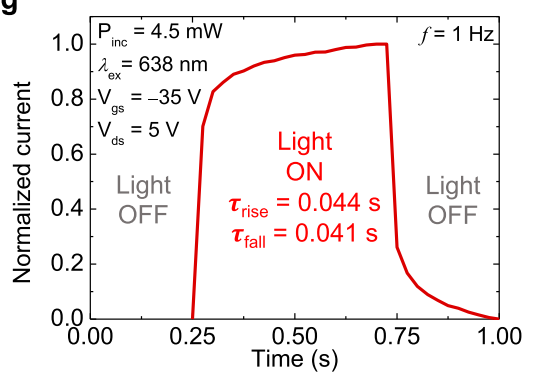

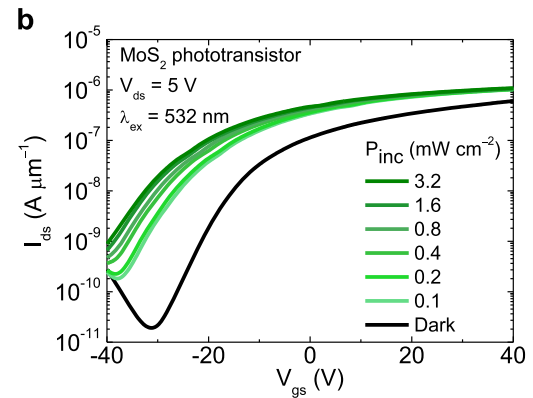

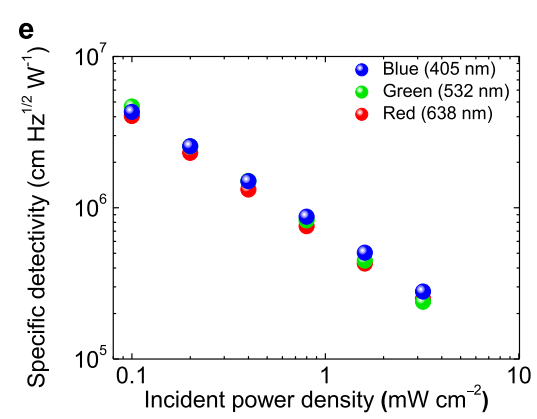

h

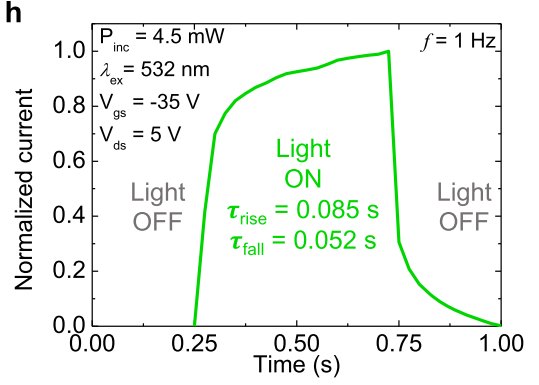

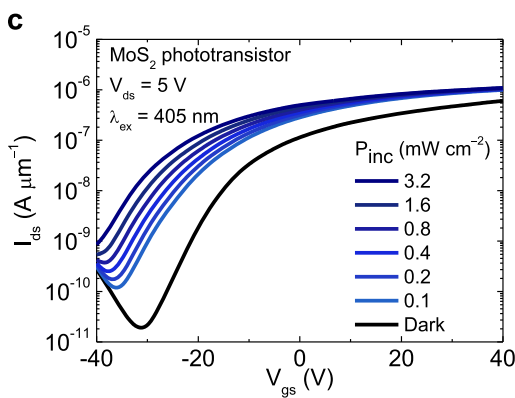

$\mathbf{f}$
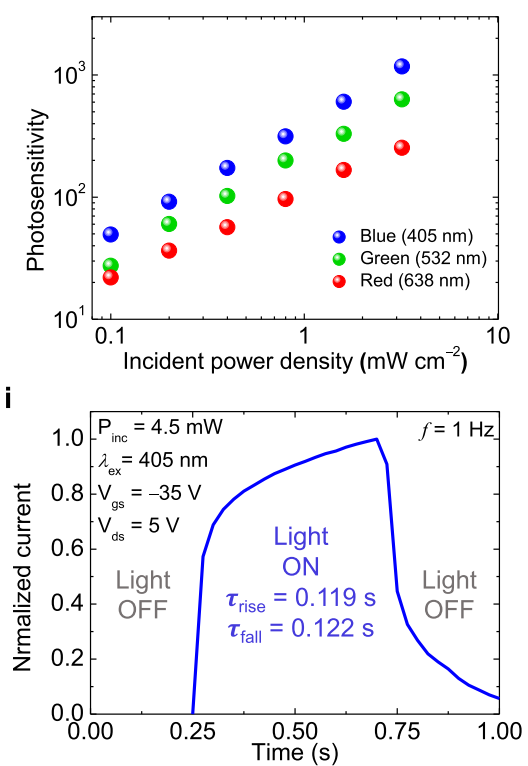

Fig. 5 Photoresponsive characteristics of a bilayer $\mathbf{M o S}_{\mathbf{2}}$ phototransistor in the image sensor array. $I_{\mathrm{ds}}-V_{\mathrm{gs}}$ curves of a transparent top-gate phototransistor based on bilayer $\mathrm{MoS}_{2}$ channel and IZO top-gate at $V_{\mathrm{ds}}=5 \mathrm{~V}$ under $\mathbf{a}$ red, $\mathbf{b}$ green, and $\mathbf{c}$ blue light illumination with various incident power densities ( $\lambda_{\mathrm{ex}}=638 \mathrm{~nm}(\mathrm{R}), 532 \mathrm{~nm}(\mathrm{G})$, and $405 \mathrm{~nm}(\mathrm{~B})$, and $P_{\mathrm{inc}}=0.1,0.2,0.4,0.8,1.6,3.2 \mathrm{~mW} \mathrm{~cm}^{-2}$ ). d Photoresponsivity, e specific detectivity, and f photosensitivity of the $\mathrm{MoS}_{2}$ phototransistor under R, G, B light illumination calculated from Fig. 4a-c. g-i Photoswitching characteristics of the MoS 2 phototransistor under temporal light illumination with $\lambda_{\mathrm{ex}}=638,532$, and $405 \mathrm{~nm}$, respectively. All switching curves were measured at $V_{\mathrm{ds}}=5 \mathrm{~V}$, $V_{\mathrm{gs}}=$ $-35 \mathrm{~V}$, and $P_{\mathrm{inc}}=4.5 \mathrm{~mW} \mathrm{~cm}^{-2}$ with illumination frequency of $1 \mathrm{~Hz}$. Rise and fall times were extracted from $20 \%$ to $80 \%$ and from $80 \%$ to $20 \%$ of the maximum current, respectively.

parameters set according to the experimental values derived from Fig. 3. The device structure implemented in the simulator is schematically represented in Fig. 6a (geometrical dimensions and electrical parameters adopted in the simulations are reported in the Supplementary Note 1$)$. The simulated $I_{\mathrm{ds}}-V_{\mathrm{gs}}$ and $I_{\mathrm{ds}}-V_{\mathrm{ds}}$ curves in the dark (i.e., with no optically generated carriers) are shown in Fig. 6b, c, respectively. The $V_{\text {th }}$ of the device under no illumination is $\approx-20 \mathrm{~V}$ and was calibrated by assuming that the Fermi energy $\left(E_{\mathrm{F}}\right)$ at equilibrium is close to the conduction band edge $\left(E_{\mathrm{C}}\right)$. This was simulated by including a large doping density $\left(N_{\mathrm{D}}=5 \times 10^{19} \mathrm{~cm}^{-3}\right)$ in the $\mathrm{MoS}_{2}$ channel.

The simulated $I_{\mathrm{ds}}-V_{\mathrm{gs}}$ curves under different light illumination conditions (each corresponding to a specific incident power density, $\left.P_{\text {inc }}\right)$ are shown in Fig. 6d. As it can be noticed, simulations correctly anticipate the large photoresponsivity observed experimentally (see Fig. 6e), which is attributed to the negative $V_{\text {th }}$ shift as shown in Fig. 6f. This behavior was reproduced by including a defect level at $0.2 \mathrm{eV}$ from the valence band edge $\left(E_{\mathrm{V}}\right)$, mimicking the narrow distribution of states above the VBM in the bandgap (see Fig. 3k). The trap state is of the donor-like type, i.e., positively charged (neutral) when filled (empty) of holes. As these traps are $0.2 \mathrm{eV}$ above $E_{\mathrm{V}}$, they tend to become easily filled with holes, the latter being provided by light. When traps capture the optically generated holes, they become positively charged and thus cause $V_{\text {th }}$ to decrease (Fig. 6f), in turn leading to the high $R_{\mathrm{ph}}$ (Fig. 6e) as also observed in the experiments (Fig. 5d). Interestingly, when performing simulations without including the trap states, the observed current modulation was relatively weak and inconsistent with experimental results. For example, Fig. $6 \mathrm{~d}$ shows the simulated $I_{\mathrm{ds}}-V_{\mathrm{gs}}$ without traps (gray lines, shifted to the right for better readability). In this case, no appreciable $V_{\text {th }}$ shift is observed as shown in Fig. 6f. This leads to a much lower $R_{\mathrm{ph}}$, as shown Fig. 6e. Figure $6 \mathrm{~g}$ shows the trapped charge density $\left(N_{T}^{+}\right)$at $V_{\mathrm{gs}}=-20 \mathrm{~V}$ in the dark and with light illumination, clearly indicating that more holes are trapped in the latter case than in the former. Figure 6h,i show the simulated band diagrams corresponding to dark and with light illumination conditions with and without the inclusion of the trap states, respectively. The comparison of the two cases shows the high conductivity modulation occurring in the simulation with the inclusion of traps (Fig. 6h) and the weak variation occurring in the case without traps (Fig. 6i). All these results corroborate the hypothesis of PG effect being responsible for high $R_{\mathrm{ph}}$.

Image sensing of bilayer $\mathrm{MoS}_{2}$ image sensor array. The photoinduced transfer characteristics of all the 64 phototransistors were measured under a light illumination with a $\lambda_{\mathrm{ex}}$ of 638,532 , 

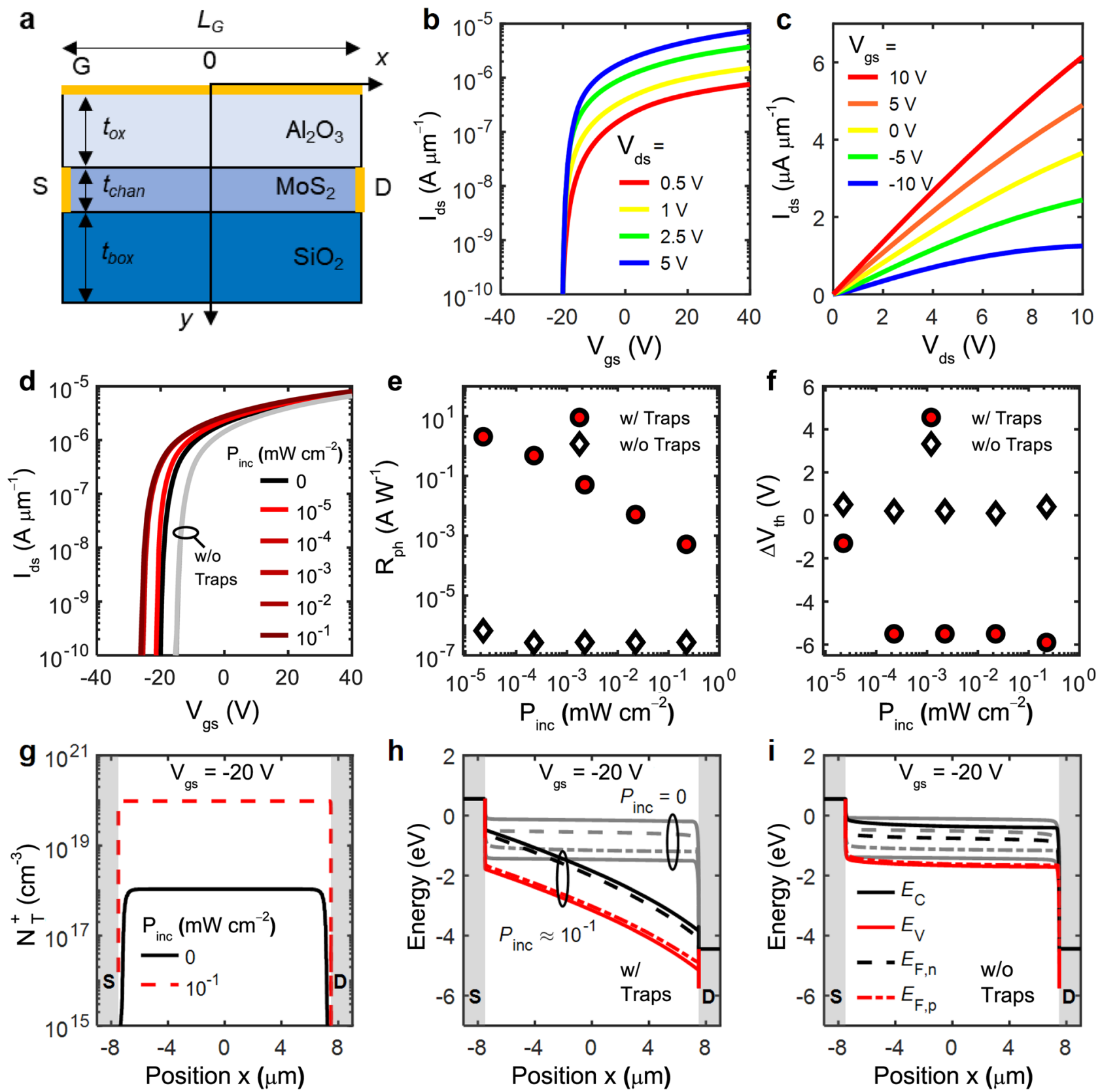

Fig. 6 Simulation results. a Sketch of the simulated $2 D$ device. $L_{G}, t_{\mathrm{ox}}, t_{\mathrm{chan},} t_{\mathrm{box}}, \mathrm{S}, \mathrm{D}$, and $\mathrm{G}$ are gate length, gate oxide thickness, MoS ${ }_{2}$ channel thickness, buried oxide thickness, source, drain, and gate, respectively. b, c Simulated $I_{\mathrm{ds}}-V_{\mathrm{gs}}$ and $I_{\mathrm{ds}}-V_{\mathrm{ds}}$ curves in the dark. d Simulated $I_{\mathrm{ds}}-V_{\mathrm{gs}}$ under light illumination, for different incident power densities $\left(P_{\text {inc }}\right)$. Simulated $I_{\mathrm{ds}}-V_{\mathrm{gs}}$ curves without traps are also included for comparison (gray lines, shifted to the right for better visibility). e, f Photoresponsivity $\left(R_{\mathrm{ph}}\right)$ and Threshold Voltage Shift $\left(\Delta V_{\mathrm{th}}\right)$ vs. $P_{\text {inc }}$ extracted from the $I_{\mathrm{ds}}-V_{\mathrm{gs}}$ simulation with and without traps, showing the correlation between high $R_{\mathrm{ph}}$ and negative $\Delta V_{\mathrm{th}} . \mathbf{g}$ Trapped charge density $\left(N_{T}^{+}\right)$at $V_{\mathrm{gs}}=-20 \mathrm{~V}$ in the dark $\left(P_{\text {inc }}=0 \mathrm{~mW} \mathrm{~cm}-2\right.$, black line) and under light illumination $\left(P_{\text {inc }} \approx 10^{-1} \mathrm{~mW} \mathrm{~cm}^{-2}\right.$, red-dashed line). $\mathbf{h}$, i Simulated energy band diagrams at $V_{\mathrm{gs}}=-20 \mathrm{~V}$ in the dark $\left(P_{\mathrm{inc}}=0 \mathrm{~mW}\right.$ $\mathrm{cm}^{-2}$, gray lines) and under light illumination $\left(P_{\mathrm{inc}} \approx 10^{-1} \mathrm{~mW} \mathrm{~cm}-2\right.$, black and red lines) with $(\mathbf{h})$ and without traps (i). $X$-axis in $\mathbf{g}$-i is the position along the channel from source to drain contacts (indicated by the shaded gray bands).

and $405 \mathrm{~nm}$ at a fixed $P_{\text {inc }}$ of $3.2 \mathrm{~mW} \mathrm{~cm}^{-2}$, to investigate the photoresponse uniformity of the active pixel image sensor array. Figure $7 \mathrm{a}-\mathrm{c}$ show the extracted photocurrent mapping of the $8 \times$ 8 active pixel image sensor array achieved using the photoinduced transfer characteristics of the 64 phototransistors under RGB light illumination. Consequently, it is confirmed that the active pixel image sensor array has a high photoresponse uniformity for all the RGB light illumination conditions. The cross-talk characteristics between adjacent pixels were also investigated, resulting in negligible cross-talk between adjacent pixels (Supplementary
Fig. 11). Supplementary Fig. 12 shows the grayscale variation under various incident power densities. Moreover, a designed turtle stencil (total $24 \times 24$ pixels) was prepared and patterned using a laser cutting system as shown in Fig. $7 \mathrm{~d}$ and Supplementary Video 1 , to investigate the image-sensing characteristics of the $8 \times 8$ active pixel image sensor array. The stencil separated into nine pieces (Fig. 7e) is sequentially placed on the active pixel image sensor array during light projection (Fig. 7f). The measurement method using the light stencil projection is described in detail in Fig. $7 \mathrm{~g}$ and the "Methods" section. Figure $7 \mathrm{~h}$ shows the 

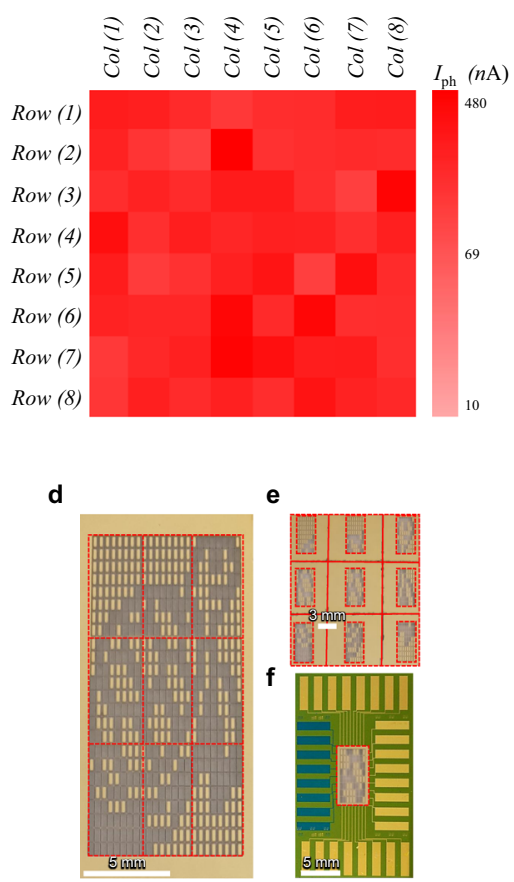

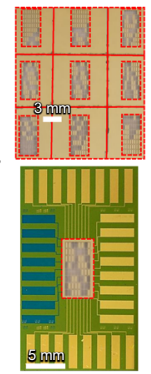

b

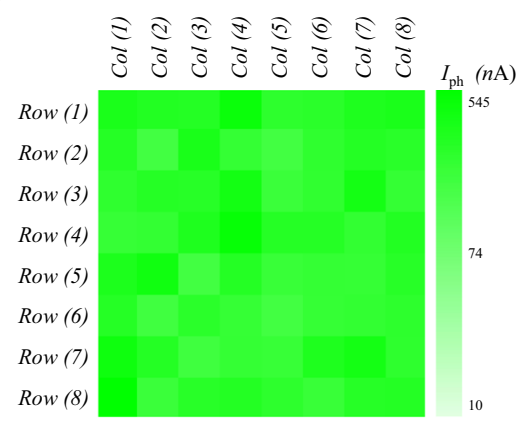

c

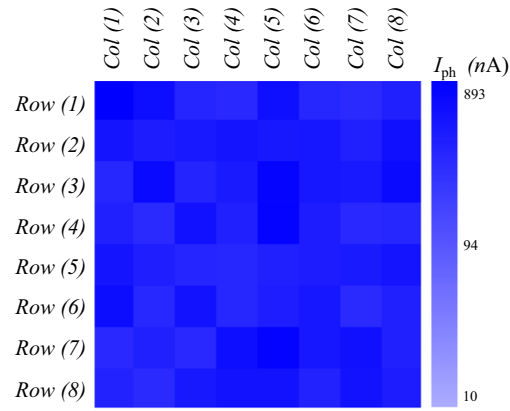

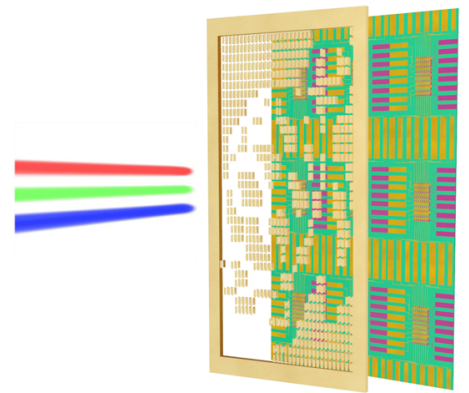

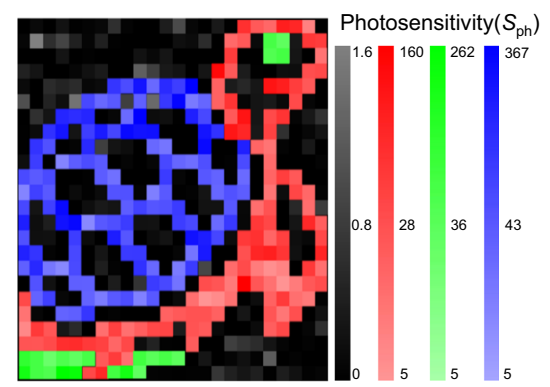

Fig. 7 Image-sensing characteristics of $8 \times 8$ bilayer $\mathbf{M o S}_{\mathbf{2}}$ image sensor array. a-c Photocurrent mapping of $64 \mathrm{MoS}_{2}$ phototransistors at $V_{\mathrm{ds}}=1 \mathrm{~V}, V_{\mathrm{gs}}$ $=-10 \mathrm{~V}$ under RGB light illumination $\left(\lambda_{\mathrm{ex}}=638,532\right.$, and $405 \mathrm{~nm}$ and $P_{\mathrm{inc}}=3.2 \mathrm{~mW} \mathrm{~cm}^{-2}$ ), indicating uniform photocurrent photoresponses. $\mathbf{d}$ Photograph of the designed turtle stencil for projection (total $24 \times 24$ pixels). e Separated turtle stencils for individual light stencil projection on $8 \times 8$ image sensor array. $\mathbf{f}$ Top view of the image sensor array covered with a piece of separated turtle stencils. $\mathbf{g}$ Measurement concept using the light stencil projection for image detection of the image sensor array. The $8 \times 8$ bilayer $\mathrm{MoS}_{2}$ image sensor array is placed behind turtle stencil and measured electrical properties under RGB light illumination $\left(\lambda_{\mathrm{ex}}=638,532\right.$, and $\left.405 \mathrm{~nm}\right)$. h Photosensitivity mapping result extracted from image detection of bilayer MoS ${ }_{2}$ image sensor array.

photosensitivity mapping result of the active pixel image sensor array obtained through the light stencil projection, indicating successful turtle image sensing (the image pixel resolution is 576 pixels). This is due to the unique pixel structure in the active pixel image sensor array composed of opaque top-gate switching transistors and transparent top-gate phototransistors. As the topgate electrode and block layer, the Au film covers the switching transistor in the pixels to remove the photocurrent interference of the switching transistor by light illumination during the image sensing through the active matrix as shown in Fig. 1. As mentioned earlier, the IZO top-gate electrode of the phototransistors enables light detection by the image sensor due to its transparent property.

Moreover, typical image signal process of complementary metal-oxide-semiconductor image sensor can be simply described as follows. First, the incident light is converted into current by the image sensor array and then read out by integrated circuit; then, current is converted to a digital signal by analog-to-digital converter. After that, the digital values are transferred into image signal processor for image processing such as image quality improvement. Finally, image data are acquired through the input/ output interface ${ }^{62,63}$.

\section{Discussion}

We demonstrated an active pixel image sensor array based on a bilayer $\mathrm{MoS}_{2}$ film. A large-area bilayer $\mathrm{MoS}_{2}$ film was directly synthesized on a $\mathrm{SiO}_{2} / \mathrm{Si}$ substrate via a two-step growth method without any transfer process. In particular, the active pixel image sensor array architecture comprised opaque top-gate switching transistors and transparent top-gate phototransistors achieved higher photoresponsive characteristics than those of photodetector without gate terminal. The main mechanism for the high $R_{\mathrm{ph}}$ of the bilayer $\mathrm{MoS}_{2}$ phototransistor is PG effect by the holes trapped at subgap states. This was confirmed by detailed, experimentally guided, numerical simulations that highlighted how the large photoresponsivity increase is a consequence of photo-excited hole trapping at subgap states close to the valence band edge. The 64 individual pixels in the $8 \times 8$ active pixel image sensor array successfully functioned under RGB light illumination. The desired photoresponsive performance and unique architecture of the proposed active pixel image sensor array can facilitate its use in next-generation image detection applications, such as ultra-thin image sensors, transparent image sensors, artificial-intelligence photosensors, and selective light-detecting imagers.

\section{Methods}

Growth of $\mathbf{M o S}_{\mathbf{2}}$ via the two-step method. A bilayer $\mathrm{MoS}_{2}$ film was synthesized using a two-step method, consisting of RF magnetron sputtering and thermal CVD. $P$-type doped Si substrates covered with $300 \mathrm{~nm}$-thick $\mathrm{SiO}_{2}$ were used as the substrates. Prior to the deposition of $\mathrm{MoS}_{2}$ via sputtering, the $\mathrm{SiO}_{2} / \mathrm{Si}$ substrates were ultrasonically cleaned for $10 \mathrm{~min}$ each in acetone, isopropyl alcohol, and deionized water. A $50.8 \mathrm{~mm}$-diameter $\mathrm{MoS}_{2}$ target (99.9\%) was used in the magnetron sputtering system. The chamber was maintained below the base pressure of $3 \times 10^{-6}$ Torr after loading the substrates and at the working pressure of $10 \mathrm{mTorr}$ with an Ar flow of 75 s.c.c.m. The $\mathrm{MoS}_{2}$ target was pre-sputtered for $10 \mathrm{~min}$ before the deposition, to remove the oxide layer on it and to make the plasma stable. The thin $\mathrm{MoS}_{2}$ films were deposited on the $\mathrm{SiO}_{2} / \mathrm{Si}$ substrates at room temperature with the RF power of $50 \mathrm{~W}$ for $14 \mathrm{~s}$.

The as-deposited $\mathrm{MoS}_{2}$ film on the $\mathrm{SiO}_{2} / \mathrm{Si}$ substrate was sulfurized in a 2 inch CVD chamber. The chamber was pumped to a low vacuum, and then Ar was injected with a flow rate of 50 s.c.c.m. For the growth of $\mathrm{MoS}_{2}$, the temperature was ramped up to $750{ }^{\circ} \mathrm{C}$ in $30 \mathrm{~min}$ and maintained for $15 \mathrm{~min}$. A gas mixture of $\mathrm{H}_{2}(5$ s.c.c.m.) and $\mathrm{H}_{2} \mathrm{~S}$ (5 s.c.c.m.) was injected into the chamber when the temperature reached $300{ }^{\circ} \mathrm{C}$. Subsequently, an annealing process was performed at $1000{ }^{\circ} \mathrm{C}$ for $1 \mathrm{~h}$ under an $\mathrm{Ar}$ (50 s.c.c.m.) and $\mathrm{H}_{2} \mathrm{~S}$ ( 5 s.c.c.m.) atmosphere. The furnace was rapidly cooled to room temperature under an Ar flow at a rate of 50 s.c.c.m. 
$\mathbf{M o S}_{\mathbf{2}}$ phototransistor fabrication. The $\mathrm{MoS}_{2}$ film was deposited on the $\mathrm{SiO}_{2} / \mathrm{Si}$ substrate for $14 \mathrm{~s}$ via RF magnetron sputtering, and then sulfurized at $750{ }^{\circ} \mathrm{C}$ and successively annealed at $1000^{\circ} \mathrm{C}$ for $1 \mathrm{~h}$ in the CVD chamber, to fabricate the active pixel image sensor array with the bilayer $\mathrm{MoS}_{2}$ film. Conventional photolithography was used to pattern the $\mathrm{MoS}_{2}$ channel. A photoresist was spin-coated for $30 \mathrm{~s}$ at 3000 r.p.m. on the $\mathrm{MoS}_{2}$ film. Afterward, the coated photoresist layer was exposed to UV light for $1 \mathrm{~s}$ and removed with a developer, which covered only the $\mathrm{MoS}_{2}$ channel. The uncovered $\mathrm{MoS}_{2}$ film was etched with $\mathrm{O}_{2}$ plasma at the power of $10 \mathrm{~W}$ for $10 \mathrm{~s}$. The photoresist remaining on the $\mathrm{MoS}_{2}$ film was removed by spraying acetone. A lift-off resist and the photoresist were sequentially coated using a spin-coater for $45 \mathrm{~s}$ at 2000 r.p.m. and $30 \mathrm{~s}$ at 3000 r.p.m., respectively. Following that, they were removed via exposure to UV light. Afterward, the $\mathrm{MoS}_{2}$ film was subjected to development processes to pattern electrodes. Subsequently, $10 \mathrm{~nm}$ titanium and $50 \mathrm{~nm}$ gold were deposited as electrodes via electron-beam evaporation and patterned via a lift-off process using photoresist remover. An 80 nm-thick $\mathrm{Al}_{2} \mathrm{O}_{3}$ as the top-gate insulator was then deposited on the lifted-off sample by ALD at $100^{\circ} \mathrm{C}$. Subsequently, IZO was deposited as the top-gate electrodes using a sputtering system. For the chemical etching of the IZO film after patterning, the sample was immersed in buffered oxide etching for $20 \mathrm{~s}$. After patterning the transparent top-gate electrodes, $\mathrm{Ti} / \mathrm{Au}(20 / 50 \mathrm{~nm})$ were deposited by electron-beam evaporation and pattern by the lift-off process as the opaque topgate electrodes. Finally, via holes were patterned using the aforementioned conventional photolithography and chemical etching processes sequentially.

Characterization of $\mathbf{M o S}_{\mathbf{2}}$. The surface of the $\mathrm{MoS}_{2}$ film grown on the $\mathrm{SiO}_{2} / \mathrm{Si}$ substrates was observed using optical microscopy (BX51M, Olympus, Co.) The heights of the bilayer $\mathrm{MoS}_{2}$ films were measured using atomic force microscopy (XE7, PSIA Co.) in non-contact mode. Raman and PL spectra were obtained using a micro-Raman spectrometer system (ALPHA300, WITec, Co.) with an excitation laser at $532 \mathrm{~nm}$ at the MEMS.Sensor Platform Center of SungKyunKwan University (SKKU). XPS (Theta Probe AR-XPS System, ThermoFisher Scientific) measurements were performed with $\mathrm{Al} \mathrm{Ka} \mathrm{X-ray} \mathrm{radiation}(1486.6 \mathrm{eV})$. The working pressure in the ultra-high-vacuum chamber during the measurement was maintained below $3 \times 10^{-9}$ mbar. C $1 s$ at $284.5 \mathrm{eV}$ was used for the calibration of the binding energies. The atomic image of $\mathrm{MoS}_{2}$ was characterized using TEM (JEM ARM 200F, JEOL). The cross-sectional TEM imaging samples were fabricated using a focused ion beam system (NX2000, HITACHI). The electrical and photoresponse properties of the $\mathrm{MoS}_{2}$ active pixel image sensor array were characterized using a semiconductor characterization system (4200 SCS, Keithley) and multi-channel fiber-coupled laser source (MCLS1, Thorlabs) in an ambient condition. The noise current of the $\mathrm{MoS}_{2}$ phototransistor was measured using a lock-in amplifier (SR830 lock-in amplifier, Stanford Research Systems). The image-sensing characteristics of the active pixel image sensor array were measured using light stencil projection. The turtle stencil $(24 \times 24$ pixels $)$ was patterned onto the golddeposited glass using a laser cutting system. The turtle stencil separated into nine pieces $(8 \times 8$ pixels) was sequentially placed on the $8 \times 8$ active pixel image sensor array. Subsequently, the individual pixels were measured by connecting the contact pads adjacent to the edge of the substrate under a light projection. To obtain the colored image, each pixel of the image sensor array was measured with the turtle stencil under RGB light that differs from pixel to pixel, respectively. The light stencil projection method was inspired and developed from previous reports ${ }^{30,64}$. Lee et al. ${ }^{30}$ reported a single-pixel imager and the image scanning system with three different light sources (RGB).

Simulation methods. The $2 \mathrm{D}$ numerical simulations model the carrier transport problem with the drift-diffusion formalism (see Supplementary Note 1 for the full equation set). This way, Poisson equation and continuity equations are solved selfconsistently for each applied bias (in terms of gate-to-source and drain-to-source voltage), to obtain the electrostatic potential and the carriers' concentration. For simplicity, the light source is considered ideal, i.e., it provides constant electron-hole pair generation rates. Shockley-Read-Hall formalism was adopted to model trap capture and emission dynamics.

\section{Data availability}

The data that support the findings of this study are available from the corresponding author upon reasonable request.

Received: 25 February 2021; Accepted: 10 May 2021; Published online: 11 June 2021

\section{References}

1. Xia, F., Wang, H., Xiao, D., Dubey, M. \& Ramasubramaniam, A. Twodimensional material nanophotonics. Nat. Photonics 8, 899-907 (2014).

2. Nourbakhsh, A., Zubair, A., Joglekar, S., Dresselhaus, M. \& Palacios, T. Subthreshold swing improvement in $\mathrm{MoS}_{2}$ transistors by the negative- capacitance effect in a ferroelectric Al-doped- $\mathrm{HfO}_{2} / \mathrm{HfO}_{2}$ gate dielectric stack. Nanoscale 9, 6122-6127 (2017).

3. Rhyee, J.-S. et al. High-mobility transistors based on large-area and highly crystalline CVD-grown $\mathrm{MoSe}_{2}$ films on insulating substrates. Adv. Mater. 28, 2316-2321 (2016).

4. Wang, Q. H., Kalantar-Zadeh, K., Kis, A., Coleman, J. N. \& Strano, M. S. Electronics and optoelectronics of two-dimensional transition metal dichalcogenides. Nat. Nanotechnol. 7, 699-712 (2012).

5. Kaasbjerg, K., Thygesen, K. S. \& Jacobsen, K. W. Phonon-limited mobility in n-type single-layer $\mathrm{MoS}_{2}$ from first principles. Phys. Rev. B 85, 115317 (2012).

6. Roy, T. et al. Field-effect transistors built from all two-dimensional material components. ACS Nano 8, 6259-6264 (2014).

7. Kim, S. et al. High-mobility and low-power thin-film transistors based on multilayer $\mathrm{MoS}_{2}$ crystals. Nat. Commun. 3, 1-7 (2012).

8. Yin, Z. et al. Single-layer $\mathrm{MoS}_{2}$ phototransistors. ACS Nano 6, 74-80 (2012).

9. Choi, W. et al. High-detectivity multilayer $\mathrm{MoS}_{2}$ phototransistors with spectral response from ultraviolet to infrared. Adv. Mater. 24, 5832-5836 (2012).

10. Li, D. et al. Two-dimensional non-volatile programmable $\mathrm{p}-\mathrm{n}$ junctions. Nat. Nanotechnol. 12, 901-906 (2017).

11. Ling, X. et al. Parallel stitching of 2D materials. Adv. Mater. 28, 2322-2329 (2016).

12. Cheng, R. et al. High-performance, multifunctional devices based on asymmetric van der Waals heterostructures. Nat. Electron. 1, 356-361 (2018).

13. Resta, G. V. et al. Doping-free complementary logic gates enabled by twodimensional polarity-controllable transistors. ACS Nano 12, 7039-7047 (2018).

14. Wang, M. et al. Robust memristors based on layered two-dimensional materials. Nat. Electron. 1, 130-136 (2018).

15. Cheng, R. et al. Few-layer molybdenum disulfide transistors and circuits for high-speed flexible electronics. Nat. Commun. 5, 1-9 (2014).

16. Wang, H. et al. Integrated circuits based on bilayer $\mathrm{MoS}_{2}$ transistors. Nano Lett. 12, 4674-4680 (2012).

17. Naqi, M. et al. Ultrasensitive multilayer $\mathrm{MoS}_{2}$-based photodetector with permanently grounded gate effect. Adv. Electron. Mater. 6, 1901256 (2020).

18. Wang, L. et al. Electronic devices and circuits based on wafer-scale polycrystalline monolayer $\mathrm{MoS}_{2}$ by chemical vapor deposition. Adv. Electron. Mater. 5, 1900393 (2019).

19. Polyushkin, D. K. et al. Analogue two-dimensional semiconductor electronics. Nat. Electron. 3, 486-491 (2020).

20. Woo, Y. et al. Large-area CVD-grown $\mathrm{MoS}_{2}$ driver circuit array for flexible organic light-emitting diode display. Adv. Electron. Mater. 4, 1800251 (2018).

21. Jayachandran, D. et al. A low-power biomimetic collision detector based on an in-memory molybdenum disulfide photodetector. Nat. Electron. 3, 646-655 (2020).

22. Choi, M. et al. Full-color active-matrix organic light-emitting diode display on human skin based on a large-area $\mathrm{MoS}_{2}$ backplane. Sci. Adv. 6, eabb5898 (2020).

23. Zhang, T. et al. Fast-response inverter arrays built on wafer-scale $\mathrm{MoS}_{2}$ by atomic layer deposition. Phys. Stat. Solid. RRL 13, 1900018 (2019).

24. Choi, C. et al. Human eye-inspired soft optoelectronic device using highdensity $\mathrm{MoS}_{2}$-graphene curved image sensor array. Nat. Commun. 8, 1-11 (2017).

25. Jang, H. et al. An atomically thin optoelectronic machine vision processor. Adv. Mater. 32, 2002431 (2020).

26. Lee, W. et al. High-resolution spin-on-patterning of perovskite thin films for a multiplexed image sensor array. Adv. Mater. 29, 1702902 (2017).

27. Goossens, S. et al. Broadband image sensor array based on graphene-CMOS integration. Nat. Photonics 11, 366-371 (2017).

28. Wong, W. S., Raychaudhuri, S., Lujan, R., Sambandan, S. \& Street, R. A. Hybrid Si nanowire/amorphous silicon FETs for large-area image sensor arrays. Nano Lett. 11, 2214-2218 (2011)

29. Lopez-Sanchez, O., Lembke, D., Kayci, M., Radenovic, A. \& Kis, A. Ultrasensitive photodetectors based on monolayer $\mathrm{MoS}_{2}$. Nat. Nanotechnol. 8, 497-501 (2013)

30. Lee, Y. T. et al. High-performance $2 \mathrm{D} \mathrm{MoS}$ phototransistor for photo logic gate and image sensor. ACS Photonics 5, 4745-4750 (2018).

31. Kim, T.-Y. et al. Transparent large-area $\mathrm{MoS}_{2}$ phototransistors with inkjetprinted components on flexible platforms. ACS Nano 11, 10273-10280 (2017).

32. Hong, S. et al. Sensory adaptation and neuromorphic phototransistors based on $\mathrm{CsPb}\left(\mathrm{Br}_{1-\mathrm{x}} \mathrm{I}_{\mathrm{x}}\right)_{3}$ perovskite and $\mathrm{MoS}_{2}$ hybrid structure. ACS Nano 14, 9796-9806 (2020).

33. Park, H. et al. Exceptionally uniform and scalable multilayer $\mathrm{MoS}_{2}$ phototransistor array based on large-scale $\mathrm{MoS}_{2}$ grown by RF sputtering, electron beam irradiation, and sulfurization. ACS Appl. Mater. Interfaces 12, 20645-20652 (2020)

34. Xu, J., Zhang, J., Zhang, W. \& Lee, C.-S. Interlayer nanoarchitectonics of twodimensional transition-metal dichalcogenides nanosheets for energy storage and conversion applications. Adv. Energy Mater. 7, 1700571 (2017). 
35. Li, H. et al. From bulk to monolayer $\mathrm{MoS}_{2}$ : evolution of Raman scattering. Adv. Funct. Mater. 22, 1385-1390 (2012).

36. Lee, C. et al. Anomalous lattice vibrations of single-and few-layer $\mathrm{MoS}_{2}$. ACS Nano 4, 2695-2700 (2010).

37. Chu, T., Ilatikhameneh, H., Klimeck, G., Rahman, R. \& Chen, Z. Electrically tunable bandgaps in bilayer $\mathrm{MoS}_{2}$. Nano Lett. 15, 8000-8007 (2015).

38. Splendiani, A. et al. Emerging photoluminescence in monolayer $\mathrm{MoS}_{2}$. Nano Lett. 10, 1271-1275 (2010).

39. Brown, N. M., Cui, N. \& McKinley, A. An XPS study of the surface modification of natural $\mathrm{MoS}_{2}$ following treatment in an RF-oxygen plasma. Appl. Surf. Sci. 134, 11-21 (1998).

40. Liu, N. et al. Large-area atomically thin $\mathrm{MoS}_{2}$ nanosheets prepared using electrochemical exfoliation. ACS Nano 8, 6902-6910 (2014).

41. Hussain, S. et al. Large-area, continuous and high electrical performances of bilayer to few layers $\mathrm{MoS}_{2}$ fabricated by RF sputtering via post-deposition annealing method. Sci. Rep. 6, 1-13 (2016).

42. Huang, F. et al. The influence of interfacial tensile strain on the charge transport characteristics of $\mathrm{MoS}_{2}$-based vertical heterojunction devices. Nanoscale 8, 17598-17607 (2016).

43. Yun, J.-M. et al. Efficient work-function engineering of solution-processed $\mathrm{MoS}_{2}$ thin-films for novel hole and electron transport layers leading to highperformance polymer solar cells. J. Mater. Chem. C 1, 3777-3783 (2013).

44. Jiao, K. et al. The role of $\mathrm{MoS}_{2}$ as an interfacial layer in graphene/silicon solar cells. Phys. Chem. Chem. Phys. 17, 8182-8186 (2015).

45. Tarasov, A. et al. Controlled doping of large-area trilayer $\mathrm{MoS}_{2}$ with molecular reductants and oxidants. Adv. Mater. 27, 1175-1181 (2015).

46. Ganatra, R. \& Zhang, Q. Few-layer $\mathrm{MoS}_{2}$ : a promising layered semiconductor. ACS Nano 8, 4074-4099 (2014).

47. Choi, M. et al. Flexible active-matrix organic light-emitting diode display enabled by $\mathrm{MoS}_{2}$ thin-film transistor. Sci. Adv. 4, eaas8721 (2018).

48. Hong, S. et al. n-Type doping effect of CVD-grown multilayer $\mathrm{MoSe}_{2}$ thin film transistors by two-step functionalization. Adv. Electron. Mater. 4, 1800308 (2018).

49. Li, T., Wan, B., Du, G., Zhang, B. \& Zeng, Z. Electrical performance of multilayer $\mathrm{MoS}_{2}$ transistors on high- $\mathrm{Al}_{2} \mathrm{O}_{3}$ coated Si substrates. AIP Adv. 5, 057102 (2015).

50. Bolshakov, P. et al. Improvement in top-gate $\mathrm{MoS}_{2}$ transistor performance due to high quality backside $\mathrm{Al}_{2} \mathrm{O}_{3}$ layer. Appl. Phys. Lett. 111, 032110 (2017).

51. Konstantatos, G. et al. Ultrasensitive solution-cast quantum dot photodetectors. Nature 442, 180-183 (2006).

52. Kim, S. et al. Interstitial Mo-assisted photovoltaic effect in multilayer $\mathrm{MoSe}_{2}$ phototransistors. Adv. Mater. 30, 1705542 (2018).

53. Buscema, M. et al. Photocurrent generation with two-dimensional van der Waals semiconductors. Chem. Soc. Rev. 44, 3691-3718 (2015).

54. Fang, H. \& Hu, W. Photogating in low dimensional photodetectors. Adv. Sci. 4, 1700323 (2017).

55. Butt, N. Z., Sarker, B. K., Chen, Y. P. \& Alam, M. A. Substrate-induced photofield effect in graphene phototransistors. IEEE Trans. Electron Devices 62, 3734-3741 (2015).

56. Furchi, M. M., Polyushkin, D. K., Pospischil, A. \& Mueller, T. Mechanisms of photoconductivity in atomically thin $\mathrm{MoS}_{2}$. Nano Lett. 14, 6165-6170 (2014).

57. Zhu, W. et al. Electronic transport and device prospects of monolayer molybdenum disulphide grown by chemical vapour deposition. Nat. Commun. 5, 1-8 (2014).

58. Früngel, F. B. Optical Pulses-Lasers-Measuring Techniques (Academic, 2014).

59. Jeon, S. et al. Gated three-terminal device architecture to eliminate persistent photoconductivity in oxide semiconductor photosensor arrays. Nat. Mater. 11, 301-305 (2012).

60. Ahn, S. E. et al. Metal oxide thin film phototransistor for remote touch interactive displays. Adv. Mater. 24, 2631-2636 (2012).
61. Sun, M. et al. Gate stimulated high-performance $\mathrm{MoS}_{2}-\mathrm{In}(\mathrm{OH})_{\mathrm{x}} \mathrm{Se}$ phototransistor. Nanotechnology 31, 095203 (2019).

62. Gove, R. J. High Performance Silicon Imaging (Elsevier, 2020).

63. Zhu, H. et al. in 2018 IEEE Asia Pacific Conference on Circuits and Systems (APCCAS) 528-531 (IEEE).

64. Choi, H. T. et al. Zero-dimensional PbS quantum dot-InGaZnO film heterostructure for short-wave infrared flat-panel imager. ACS Photonics 7, 1932-1941 (2020)

\section{Acknowledgements}

This research was supported in part by the National Research Foundation of Korea (2021R1A2B5B02002167, 2021M3H4A1A02056037, 2020H1D3A2A02103378, and 2020R1I1A1A01070907)

\section{Author contributions}

S.K. designed and supervised this project. S.H. performed all the experiments, except for the synthesis of $\mathrm{MoS}_{2}$. S.C. performed the synthesis of $\mathrm{MoS}_{2}$ and contributed to the experiments and spectroscopic analysis. S.B. and A.B. contributed to the synthesis of $\mathrm{MoS}_{2}$. N.L. and H.Y. performed the spectroscopic analysis of $\mathrm{MoS}_{2}$. B.H.K. and H.J.K. performed the IZO deposition. H.J.Y. performed the UPS measurement. N.Z. and M.A.A conceived and performed the device modeling and numerical simulations. All the authors analyzed the data. S.H., N.Z., S.C., N.L., and S.K. wrote the manuscript. All the authors have approved the final version of the manuscript.

\section{Competing interests}

The authors declare no competing interests.

\section{Additional information}

Supplementary information The online version contains supplementary material available at https://doi.org/10.1038/s41467-021-23711-x.

Correspondence and requests for materials should be addressed to M.A.A. or S.K

Peer review information Nature Communications thanks Xiaomu Wang, Do Kyung Hwang, and the other anonymous reviewers for their contribution to the peer review of this work.

Reprints and permission information is available at http://www.nature.com/reprints

Publisher's note Springer Nature remains neutral with regard to jurisdictional claims in published maps and institutional affiliations.

Open Access This article is licensed under a Creative Commons Attribution 4.0 International License, which permits use, sharing, adaptation, distribution and reproduction in any medium or format, as long as you give appropriate credit to the original author(s) and the source, provide a link to the Creative Commons license, and indicate if changes were made. The images or other third party material in this article are included in the article's Creative Commons license, unless indicated otherwise in a credit line to the material. If material is not included in the article's Creative Commons license and your intended use is not permitted by statutory regulation or exceeds the permitted use, you will need to obtain permission directly from the copyright holder. To view a copy of this license, visit http://creativecommons.org/ licenses/by/4.0/.

(C) The Author(s) 2021 